\title{
Referral offer and acceptance following supportive care problem identification in an Australian comprehensive cancer service
}

Gemma Skaczkowski ${ }^{1,2}$, Penny Sanderson ${ }^{1}$, Melissa Shand ${ }^{3}$, Amanda Byrne ${ }^{3}$, Carlene Wilson ${ }^{1,2}$ ${ }_{1}^{1}$ Olivia Newton-John Cancer Wellness \& Research Centre, Austin Health

2 School of Psychology \& Public Health, La Trobe University

3 North Eastern Melbourne Integrated Cancer Service

\section{Introduction}

- The success of screening programs in addressing patient needs depends on the quality of the triage process to relevant services.

- Australian studies report highly variable rates of referral offer post-screening, from 21\%-91\%.,

- Referral acceptance varies according to demographic factors, such as sex, education level and age..$^{2-4}$

Aim: To examine factors associated with the offer of referral to services, and subsequent acceptance.

\section{Method}

Retrospective review of patient medical records. Distress Thermometer (DT) and Problem Checklist (PC) completions from 01/01/2013-30/06/2014.

Data collected on:

- Demographic, disease and treatment information

- DT and PC scores

- Referral(s) offered?

- Referral(s) accepted/declined?

Data for each patient's first recorded DT and PC completion was analysed.

\section{$71 \%$ of referrals accepted overall}

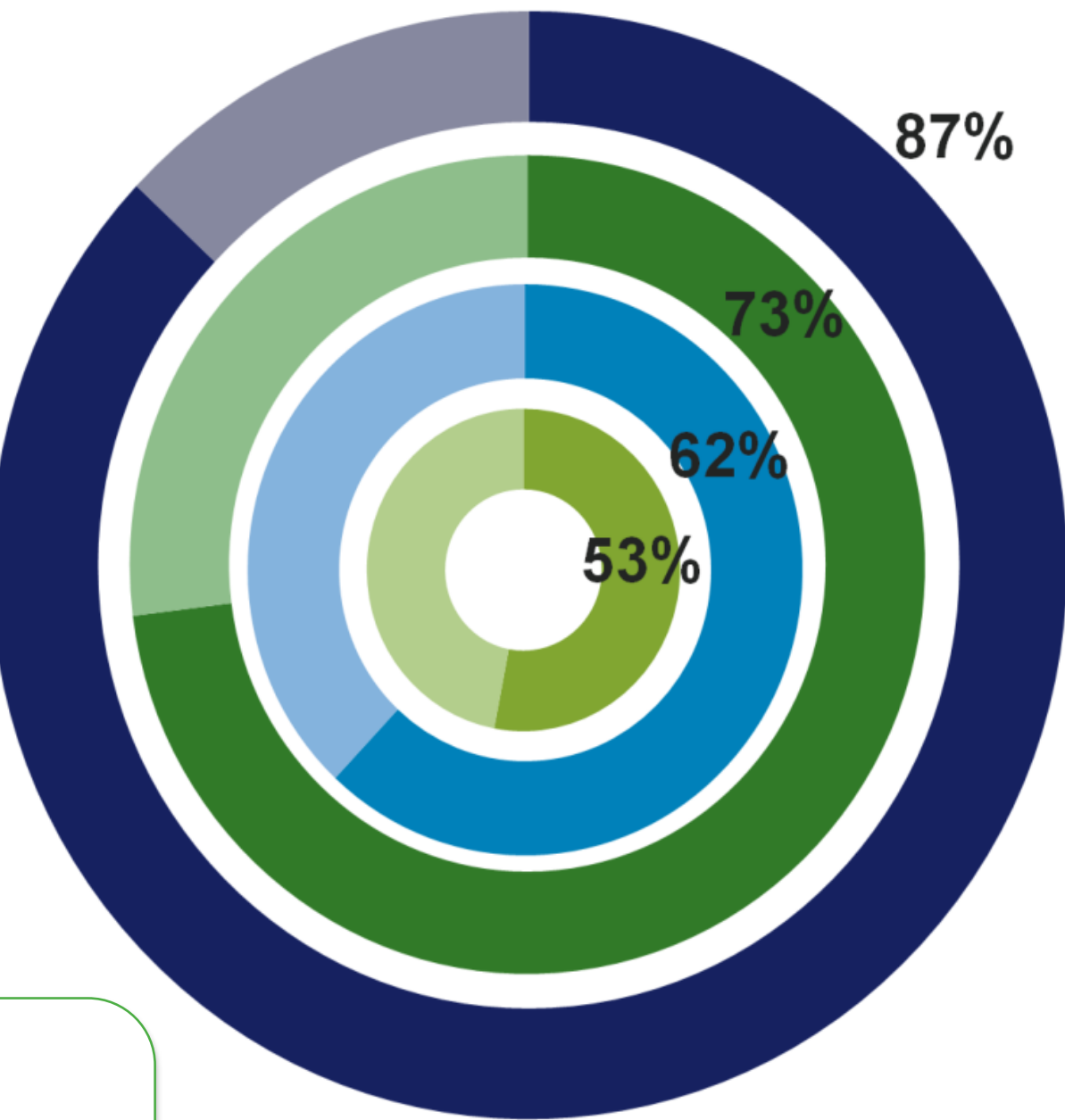

- Physical referrals

- Practical referrals

- Other referrals

- Emotional referrals

\section{Results}

Higher rates of referral offer predicted by:

- Higher distress ( $p=.025)$

- Greater number of problems $(p<.001)$

Higher rates of referral acceptance predicted by:

- Lower distress ( $p=.049)$

- Greater number of problems $(p<.001)$

- Type of referral offered $(p<.001)$

- Type of treatment $(p<.001)$

\section{3 patients}

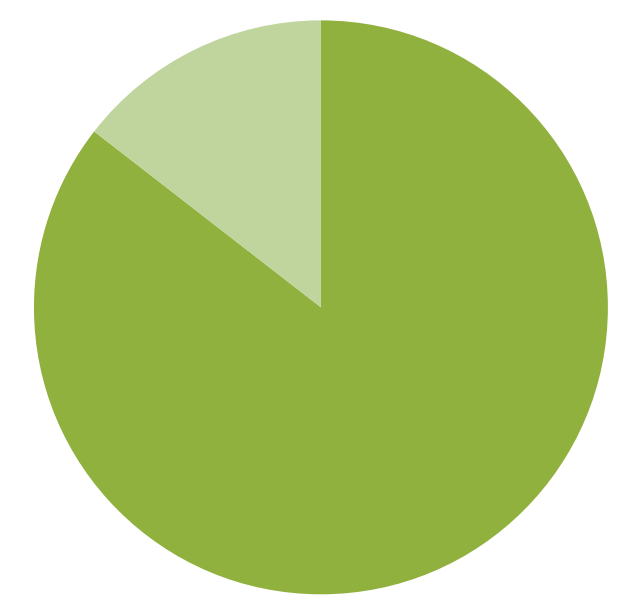

$86 \%$ accepted offer of assessment

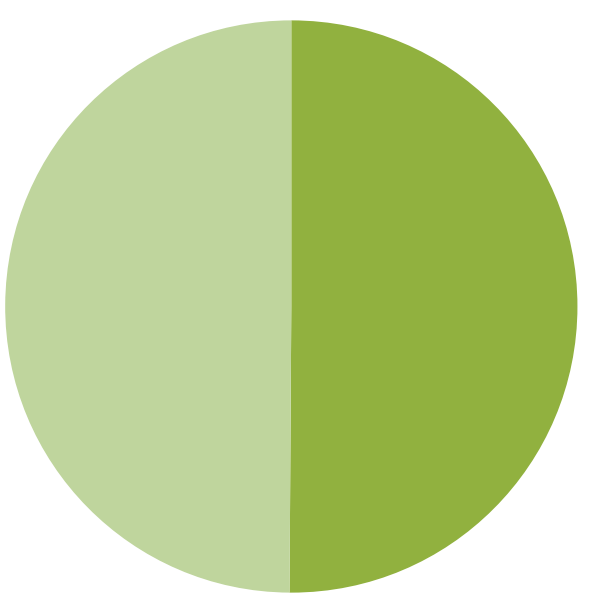

$50 \%$ reported high levels of distress

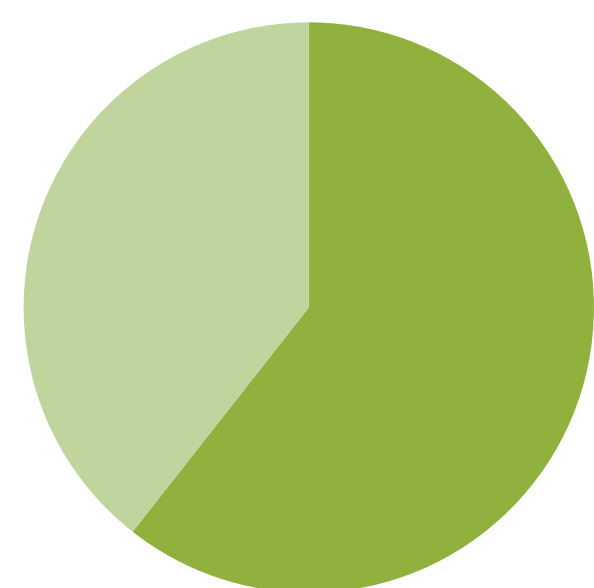

$61 \%$ were offered referrals

Conclusions

- Referral offer and acceptance influenced by level of distress and problems, not patient demographics.

- Referral acceptance influenced by the type of referral offered; physical referrals most often accepted.

- Triage to services via problem identification accurately addressed the needs of cancer patients.

- These results were found in the context of a large metropolitan hospital with an in-house supportive care service. 